\title{
Concerning Solutions of the VLF Mode Problem for an Anisotropic Curved Ionosphere
}

\author{
James R. Wait
}

\section{Contribution from Central Radio Propagation Laboratory, National Bureau of Standards, Boulder, Colo.}

(Received December 5, 1962)

\begin{abstract}
The influence of earth curvature in the theorv of reflection from the ionosphere is considered. By choosing a rather idealized model, the significance of usual earth-flattening procedures can be displaved quite readily. To simplify the analysis, it is assumed that the earth's magnetic field is vertical everywhere. It is shown that the curved ionosphere may be represented by homogeneous planar slabs, provided the local value of layer curvature is used. The results in the present paper are compared with some corresponding expressions obtained by Johler and Berry [1962].
\end{abstract}

\section{Introduction}

Boundary value problems involving magneto plasmas, such as the ionosphere, are a great deal more complicated than the corresponding problems for isotropic media. For this reason a number of gross simplifications are often made in order to achieve tractability. For example, in most of the existing literature on radio propagation it is assumed that the ionosphere may be regarded as a planar stratified medium. In fact, Budden's exhaustive treatise [Budden, 1961] is restricted entirely to this case. Also, in prior work on VLF mode theory it has been somewhat tacitly assumed that the ionosphere may be regarded, in a local sense, as a planar stratified medium. Justifications for this step are based on arguments valid only for isotropic media. Earth curvature is accounted for only in the nonionized waveguide region beneath the base of the ionosphere [Wait, 1961a, 1962; Katzin and Koo, 1962; Budden, 1962]. Since the anisotropic properties of the ionosphere play an important role in VLF mode theory, it seems that this aspect of the subject warrants some scrutiny, and this is the main purpose of the present paper.

The situation considered here is rather idealized. The ionosphere is regarded as a spherically stratified plasma with a superimposed radial magnetic field of constant strength. Such a model has been considered by van der Wijck [1946], Bremmer [1949], and more recently by Krasnushkin [1961]. If this restriction, on the magnetic field, were not made the problem would become intractable unless some other simplifying assumptions were made such as a purely transverse field [Wait, 1961b].

\section{Formulation}

Choosing a spherical coordinate system $(\theta, \phi, r)$, the base of the ionosphere can be regarded as the spherical surface $r=c$. For an ionospheric-type plasma, the dielectric constant $(\epsilon)$ has the form of a tensor. The relation between the displacement

vector $\vec{D}$ and the electric field $\vec{E}$ is then

$$
\vec{D}=(\epsilon) \vec{E},
$$

which may be written more explicitly in matrix form as

$$
\left[\begin{array}{c}
D_{\theta} \\
D_{\phi} \\
D_{r}
\end{array}\right]=\left[\begin{array}{ccc}
\epsilon_{11} & \epsilon_{12} & 0 \\
-\epsilon_{12} & \epsilon_{22} & 0 \\
0 & 0 & \epsilon_{33}
\end{array}\right]\left[\begin{array}{l}
E_{\theta} \\
E_{\phi} \\
E_{r}
\end{array}\right] .
$$

The elements of the dielectric tensor may be a function of $r$ but constant with respect to $\theta$ and $\phi$. They depend on the strength of the earth's magnetic field and on the electron density, ion density, and frequency of collisions between them. The case usually considered is that in which the electromagnetic forces influence only the electrons and the motion of the ions is neglected. In this case [Bremmer, 1949]

$$
\begin{aligned}
& \frac{\epsilon_{11}}{\epsilon_{0}}=\frac{\epsilon_{22}}{\epsilon_{0}}=1-\frac{i(\nu+i \omega) \omega_{0}^{2} / \omega}{\omega_{h}^{2}+(\nu+i \omega)^{2}}, \\
& \frac{\epsilon_{12}}{\epsilon_{0}}=\frac{i \omega_{h} \omega_{0}^{2} / \omega}{\omega_{h}^{2}+(\nu+i \omega)^{2}}, \\
& \frac{\epsilon_{33}}{\epsilon_{0}}=1-\frac{i \omega_{0}^{2}}{(\nu+i \omega) \omega},
\end{aligned}
$$


where $\omega_{0}$ is the (electron) plasma frequency, $\omega_{h}$ is the (electron) gyrofrequency, and $\nu$ is the effective collision frequency (for electrons). The generalizations of these formulae to include the influence of heavy ions and the effect of an energy-dependent collision frequency have been discussed elsewhere [Sen and Wyler, 1960; Johler, 1962; Wait, 1962]. For purposes of the present analysis, it is sufficient to point out that, even under these general conditions, the dielectric tensor has the form given by (2), provided the magnetic field is vertical. Therefore, the subsequent theory will not be restricted just to an electron plasma with constant collision frequency.

Maxwell's equations,

$$
\begin{aligned}
& \text { curl } \vec{E}=-i \mu \omega \vec{H} \text {, } \\
& \text { curl } \vec{H}=i(\epsilon) \omega \vec{E},
\end{aligned}
$$

where $(\epsilon)$ is a tensor may be written explicitly in the form

$$
\begin{gathered}
-\frac{1}{r} \frac{\partial}{\partial r}\left(r E_{\phi}\right)=-i \mu \omega H_{\theta}, \\
\frac{1}{r}\left[\frac{\partial}{\partial r}\left(r E_{\theta}\right)-\frac{\partial E_{r}}{\partial \theta}\right]=-i \mu \omega H_{\phi}, \\
\frac{1}{r \sin \theta} \frac{\partial}{\partial \theta}\left(\sin \theta E_{\phi}\right)=-i \mu \omega H_{r}, \\
-\frac{1}{r} \frac{\partial}{\partial r}\left(r H_{\phi}\right)=i \omega\left[\epsilon_{11} E_{\theta}+\epsilon_{12} E_{\phi}\right], \\
\frac{1}{r}\left[\frac{\partial}{\partial r}\left(r H_{\theta}\right)-\frac{\partial H_{r}}{\partial \theta}\right]=i \omega\left[-\epsilon_{12} E_{\theta}+\epsilon_{22} E_{\phi}\right], \\
\frac{1}{r \sin \theta} \frac{\partial}{\partial \theta}\left(\sin \theta H_{\phi}\right)=i \omega \epsilon_{33} E_{r} .
\end{gathered}
$$

In the above it has been assumed that $\partial / \partial \phi=0$.

It is now a simple matter to express the $r$ and $\theta$ components of the field in terms of the $\phi$ component. To facilitate this, two auxiliary functions $M$ and $N$ are introduced which are defined by

$$
\begin{aligned}
& E_{\phi}=\frac{1}{r} \frac{\partial M}{\partial \theta}, \\
& H_{\phi}=\frac{1}{r} \frac{\partial N}{\partial \theta} .
\end{aligned}
$$

Then, without difficulty, it follows that

$$
\begin{aligned}
-i \mu \omega H_{r} & =\frac{1}{r^{2} \sin \theta} \frac{\partial}{\partial \theta}\left(\sin \theta \frac{\partial M}{\partial \theta}\right), \\
i \epsilon_{33} \omega E_{r} & =\frac{1}{r^{2} \sin \theta} \frac{\partial}{\partial \theta}\left(\sin \theta \frac{\partial N}{\partial \theta}\right),
\end{aligned}
$$

$$
\begin{gathered}
i \mu \omega H_{\theta}=\frac{1}{r} \frac{\partial^{2} M}{\partial r \partial \theta}, \\
i \epsilon_{11} \omega E_{\theta}=-\frac{1}{r} \frac{\partial^{2} N}{\partial r \partial \theta}-\frac{i \epsilon_{12} \omega}{r} \frac{\partial M}{\partial \theta} .
\end{gathered}
$$

Furthermore, it is found that $M$ and $N$ satisfy the two coupled equations

$$
\begin{array}{r}
\epsilon_{33} r^{2} \frac{\partial}{\partial r} \frac{1}{\epsilon_{11}} \frac{\partial N}{\partial r}+k^{2}\left(\frac{\epsilon_{33}}{\epsilon_{0}}\right) r^{2} N+i \epsilon_{33} \omega r^{2} \frac{\partial}{\partial r}\left(\frac{\epsilon_{12}}{\epsilon_{11}} M\right) \\
+\frac{1}{\sin \theta} \frac{\partial}{\partial \theta}\left(\sin \theta \frac{\partial}{\partial \theta} N\right)=0 \\
\frac{-i \mu \omega \epsilon_{12}}{\epsilon_{11}} r^{2} \frac{\partial N}{\partial r}+r^{2} \frac{\partial^{2} M}{\partial r^{2}}+\frac{k^{2} r^{2}}{\epsilon_{0}}\left[\epsilon_{22}+\frac{e_{12}^{2}}{\epsilon_{11}}\right] M \\
+\frac{1}{\sin \theta} \frac{\partial}{\partial \theta}\left(\sin \theta \frac{\partial}{\partial \theta} M\right)=0
\end{array}
$$

where $k^{2}=\epsilon_{0} \mu \omega^{2}$. In the case of isotropic media, $M$ and $N$ are proportional to Debye potentials. Equations (18) and (19) are applicable in regions external to the source.

\section{Solution}

A solution of (18) and (19) is now sought in the form

$$
\begin{aligned}
& N=F(r) P_{\nu}(-\cos \theta), \\
& M=G(r) P_{\nu}(-\cos \theta),
\end{aligned}
$$

where $P_{\nu}$ is the Legendre function which has the required asymptotic behavior and has no singularities except at $\theta=0$. It is a solution of the equation

$$
\frac{1}{\sin \theta} \frac{\partial}{\partial \theta}\left[\sin \theta \frac{\partial P_{\nu}(-\cos \theta)}{\partial \theta}\right]+\nu(\nu+1) P_{\nu}(-\cos \theta)=0 \text {. }
$$

The radial functions $F$ and $G$ must then satisfy

$$
\begin{aligned}
\epsilon_{33} \frac{d}{d r}\left(\frac{1}{\epsilon_{11}} \frac{d F}{d r}\right)+k^{2} \frac{\epsilon_{33}}{\epsilon_{0}} F+i \omega \epsilon_{33} & \frac{d}{d r}\left(\frac{\epsilon_{12}}{\epsilon_{11}} G\right) \\
& -\frac{\nu(\nu+1)}{r^{2}} F=0
\end{aligned}
$$

$$
\frac{d^{2} G}{d r^{2}}+\frac{k^{2}}{\epsilon_{0}}\left[\epsilon_{22}+\frac{\epsilon_{12}^{2}}{\epsilon_{11}}\right] G-i \mu \omega \frac{\epsilon_{12}}{\epsilon_{11}} \frac{\partial F}{\partial r}-\frac{\nu(\nu+1)}{r^{2}} G=0 .
$$

In the case of isotropic media (i.e., $\epsilon_{12}=0$ ), the equations become uncoupled. As a further check, the medium may be taken to be homogeneous and isotropic (i.e., $\epsilon_{11}=\epsilon_{22}=\epsilon_{33}=\epsilon$ where $\epsilon$ is a constant). Then $F$ and $G$ satisfy the same differential equation given by

$$
\left\{\frac{d^{2}}{d r^{2}}+k^{2}\left(\frac{\epsilon}{\epsilon_{0}}\right)-\frac{\nu(\nu+1)}{r^{2}}\right\}_{G}^{F}=0 .
$$


Solutions are linear combinations of the functions $Z h_{\nu}^{(1)}(Z)$ and $Z h_{\nu}^{(2)}(Z)$, where $Z=k\left(\epsilon / \epsilon_{0}\right)^{1 / 2} r$ and where $h_{\nu}^{(1)}$ and $h_{\nu}^{(2)}$ are spherical Hankel functions. Most proponents [Budden, 1962; Katzin and Koo, 1962] of earth-flattening procedures start with an equation equivalent to (25). Then they develop the idea that the term

$$
k^{2}\left(\frac{\epsilon}{\epsilon_{0}}\right)-\frac{\nu(\nu+1)}{r^{2}}
$$

may be replaced by

$$
k^{2}\left(\frac{\epsilon_{\mathrm{eff}}(r)}{\epsilon_{0}}\right)
$$

where $\epsilon_{\text {eff }}(r)$ is the effective dielectric constant for the equivalent planar region. Unfortunately, in the anisotropic case the solutions of the coupled equations cannot be expressed in terms of spherical Hankel functions even if the medium is homogeneous. Thus, the earth flattening procedures are open to question when dealing with magnetoplasma media.

To solve the coupled wave equations for the general case, resort must be made to numerical techniques. To simplify the procedure it is suggested that the ionosphere above the surface $r=c=r_{0}$ be broken into a number of concentric homogeneous regions which are bounded by the surfaces $r_{0}, r_{1}, r_{2} \ldots r_{m-1}, r_{m} \ldots$ $r_{M}$. For example, within the $m$ th region, the elements of the dielectric tensor may be replaced bv constants. Thus, for $r_{m-1}<r<r_{m}$,

$$
\epsilon_{11}=\epsilon_{22}=\epsilon_{m}, \epsilon_{33}=\hat{\epsilon}_{m} \text { and } \epsilon_{12}=i g_{m},
$$

which corresponds to a dielectric tensor having the form

$$
(\boldsymbol{\epsilon})=\left(\begin{array}{ccc}
\boldsymbol{\epsilon}_{m} & i g_{m} & 0 \\
-i g_{m} & \epsilon_{m} & 0 \\
0 & 0 & \hat{\epsilon}_{m}
\end{array}\right)
$$

for the $m$ th region. The coupled equations now have the form

and

$$
\frac{\hat{\epsilon}_{m}}{\epsilon_{m}} \frac{d^{2} F_{m}}{d r^{2}}+k^{2} \frac{\hat{\epsilon}_{m}}{\epsilon_{0}} F_{m}-\frac{\omega g_{m} \hat{\epsilon}_{m}}{\epsilon_{m}} \frac{d G_{m}}{d r}-\lambda^{2} F_{m}=0
$$

$$
\frac{d^{2} G_{m}}{d r^{2}}+k^{2} \frac{\epsilon_{m}}{\epsilon_{0}}\left(1-\frac{g_{m}^{2}}{\epsilon_{m}^{2}}\right) G_{m}+\frac{\mu \omega g_{m}}{\epsilon_{m}} \frac{d F_{m}}{d r}-\lambda^{2} G_{m}=0,
$$

where

$$
\lambda^{2}=\frac{\nu(\nu+1)}{r^{2}}
$$

In most cases the quantity $\lambda^{2}$ can be regarded as a constant within the layer. Thus, it is replaced by

$$
\lambda^{2}=\lambda_{m}^{2}=\frac{\nu(\nu+1)}{r_{m}^{2}}
$$

for the $m$ th layer. Solutions of the coupled differential equations may now be found in the form

and

$$
F_{m}=p_{m} e^{-u_{m} r}
$$

$$
G_{m}=q_{m} e^{-u_{m} r} .
$$

Substituting these into (27) and (28) leads to the two algebraic equations

$$
u_{m}^{2} \frac{\hat{\boldsymbol{\epsilon}}_{m}}{\boldsymbol{\epsilon}_{m}} p_{m}+k^{2} \frac{\hat{\boldsymbol{\epsilon}}_{m}}{\boldsymbol{\epsilon}_{0}} p_{m}-\lambda_{m}^{2} p_{m}+\frac{\omega g_{m} \hat{\boldsymbol{\epsilon}}_{m} u_{m}}{\boldsymbol{\epsilon}_{m}} q_{m}=0
$$

and

$$
\frac{-\mu \omega g_{m} u_{m} p_{m}}{\epsilon_{m}}+u_{m}^{2} q_{m}+k^{2} \frac{\epsilon_{m}}{\epsilon_{0}}\left(1-\frac{g_{m}^{2}}{\epsilon_{m}^{2}}\right) q_{m}-\lambda_{m}^{2} q_{m}=0 .
$$

In order for a solution to exist, the determinant of the coefficients $p_{m}$ and $q_{m}$ must vanish. This condition can be written compactly in the form

where

$$
u_{m}^{4}+u_{m}^{2}\left[s_{1}^{2}+s_{2}^{2}\right]+s_{1}^{2} s_{2}^{2}-s_{2}^{2} \frac{k^{2} g_{m}^{2}}{\epsilon_{0} \epsilon_{m}}=0 .
$$

$$
\begin{gathered}
s_{2}^{2}=\frac{\boldsymbol{\epsilon}_{m}}{\hat{\boldsymbol{\epsilon}}_{m}}\left(k^{2} \frac{\hat{\epsilon}_{m}}{\epsilon_{0}}-\lambda_{m}^{2}\right), \\
s_{1}^{2}=k^{2} \frac{\boldsymbol{\epsilon}_{m}}{\epsilon_{0}}-\lambda_{m}^{2} .
\end{gathered}
$$

There are four solutions of this equation; two representing outgoing waves and two representing incoming waves. These are denoted $\pm u_{m}$ and $\pm u_{m}^{\prime}$. The equation is equivalent to the Booker quartic for plane waves in anisotropic media [Budden, 1961].

The general solution in the $m$ th layer may then be written

$$
F_{m}=\vec{p}_{m} e^{-u_{m} r}+{\overleftarrow{p_{m}}} e^{+u_{m} r}+{\overrightarrow{p_{m}^{\prime}}}^{-u_{m}^{\prime}}+\stackrel{\leftarrow}{p_{m}^{\prime}} e^{+u_{m}^{\prime} r}
$$

and

$$
G_{m}=\vec{q}_{m} e^{-u_{m} r}+\overleftarrow{q}_{m} e^{+u_{m} r}+{\overrightarrow{q_{m}^{\prime}}}_{m} e^{-u_{m}^{\prime} r}+\overleftarrow{q_{m}^{\prime}} e^{+u_{m}^{\prime} r}
$$

when the $p$ 's are arbitrary constants. The $q$ 's are related to corresponding $p$ 's through (32) or (33). Therefore, for convenience in what follows,

$$
\begin{aligned}
& \vec{q}_{m}=\alpha\left(u_{m}\right) \vec{p}_{m}, \overleftarrow{q}_{m}=\alpha\left(-u_{m}\right) \vec{p}_{m}, \\
& \vec{q}_{m}^{\prime}=\alpha\left(u_{m}^{\prime}\right) \vec{p}_{m}, \overleftarrow{q_{m}^{\prime}}=\alpha\left(-u_{m}^{\prime}\right){\overrightarrow{q_{m}^{\prime}}}^{\prime}
\end{aligned}
$$

where the function $\alpha(u)$ is known. The imaginary part of the $u_{m}^{\prime} s$ is chosen to be positive and, thus, the arrows $\rightarrow$ and $\leftarrow$ represent outgoing and ingoing waves, respectively. In the outermost region, where $m=M$, only outgoing waves are permissible and, thus, $\overleftarrow{p}_{M}=\overleftarrow{p}_{M}^{\prime}=\overleftarrow{q}_{M}=\overleftarrow{q}_{M}^{\prime}=0$. Since the tangential field components are to be continuous at the interfaces, it follows, from (12), (13), (16), and (17) that four 
continuity conditions are required at the surface $r=r_{m}$. These are

$$
\begin{gathered}
F_{m}=F_{m+1}, \\
G_{m}=G_{m+1}, \\
\frac{d G_{m}}{d r}=\frac{d G_{m+1}}{d r}, \\
\frac{1}{\epsilon_{m}}\left[\frac{d F_{m}}{d r}-g_{m} \omega G_{m}\right]=\frac{1}{\epsilon_{m+1}}\left[\frac{d F_{m+1}}{d r}-g_{m+1} \omega G_{m+1}\right] .
\end{gathered}
$$

Therefore, four independent relations between the coefficients are available at each interface from $m=1$ to $m=M$.

To obtain the complete solution of the problem it is necessary to consider the fields in the free space or waveguide region $a<r<r_{0}$. Here the fields are obtained from

$$
\begin{gathered}
E_{\phi}=\frac{1}{r} \frac{\partial M_{0}}{\partial \theta}, \\
H_{\phi}=\frac{1}{r} \frac{\partial N_{0}}{\partial \theta}, \\
-i \mu \omega H_{r}=\frac{1}{r^{2} \sin \theta} \frac{\partial}{\partial \theta}\left(\sin \theta \frac{\partial M_{0}}{\partial \theta}\right), \\
i \epsilon_{0} \omega E_{r}=\frac{1}{r^{2} \sin \theta} \frac{\partial}{\partial \theta}\left(\sin \theta \frac{\partial N_{0}}{\partial \theta}\right), \\
i \mu \omega H_{\theta}=\frac{1}{r} \frac{\partial^{2} M_{0}}{\partial r \partial \theta}, \\
i \epsilon_{0} \omega E_{\theta}=-\frac{1}{r} \frac{\partial^{2} N_{0}}{\partial r \partial \theta}
\end{gathered}
$$

which is a special case of equations (12) to (17) for isotropic media. In this case

$$
N_{0}=F_{0}(r) P_{\nu}(-\cos \theta),
$$

and

$$
M_{0}=G_{0}(r) P_{\nu}(-\cos \theta),
$$

where $G_{0}$ and $F_{0}$ satisfy

$$
\left[\frac{d^{2}}{d r^{2}}+k^{2}-\frac{\nu(\nu+1)}{r^{2}}\right] \begin{aligned}
& G_{0} \\
& F_{0}
\end{aligned}=0 .
$$

Therefore, solutions in the region $a<r<r_{0}$ are of the form

$$
\frac{F_{0}(r)}{k r}=f_{1} h_{\nu}^{(1)}(k r)+f_{2} h_{\nu}^{(2)}(k r)
$$

and

$$
\frac{G_{0}(r)}{k r}=g_{1} h_{\nu}^{(1)}(k r)+g_{2} h_{\nu}^{(2)}(k r) .
$$

The boundary conditions at the level $r=r_{0}$ are

$$
\begin{gathered}
F_{0}=F_{1} \\
G_{0}=G_{1}, \\
\frac{d G_{0}}{d r}=\frac{d G_{1}}{d r}, \\
\frac{1}{\epsilon_{0}} \frac{d F_{0}}{d r}=\frac{1}{\epsilon_{1}}\left[\frac{d F_{1}}{d r}-g_{1} \omega G_{1}\right] .
\end{gathered}
$$

For all practical purposes, the ground may be characterized by a surface admittance $Y_{g}$ and a surface impedance $Z_{g}$. Thus

and

$$
H_{\phi}=Y_{g} E_{\phi}
$$

$$
E_{\phi}=-Z_{g} H_{\phi},
$$

at $r=a$. In terms of $G_{0}$ and $F_{0}$ these may be written

$$
i \mu \omega Y_{g} G_{0}=\frac{d G_{0}}{d r},
$$

and

$$
i \epsilon_{0} \omega Z_{g} F_{0}=\frac{d F_{0}}{d r} .
$$

at $r=a$. In the case of a homogeneous ground of conductivity $\sigma_{g}$ and dielectric constant $\epsilon_{g}$,

and

$$
\begin{aligned}
Y_{g} & =\frac{1}{\eta_{0}}\left(\frac{\sigma_{g}+i \epsilon_{g} \omega}{i \epsilon_{0} \omega}\right)^{1 / 2}\left[1-\frac{i \epsilon_{0} \omega}{\sigma_{g}+i \epsilon_{g} \omega}\right]^{1 / 2}, \\
Z_{g} & =\eta_{0}\left(\frac{i \epsilon_{0} \omega}{\sigma_{g}+i \epsilon_{g} \omega}\right)^{1 / 2}\left[1-\frac{i \epsilon_{0} \omega}{\sigma_{g}+i \epsilon_{g} \omega}\right]^{1 / 2} .
\end{aligned}
$$

In most actual situations the square bracket factors in these expressions may be replaced by unity, and, furthermore, displacement currents in the ground are usually of minor significance. Thus

$$
Z_{g} \cong 1 / Y_{g} \cong\left(i \mu \omega / \sigma_{g}\right)^{1 / 2} .
$$

The boundary conditions as exemplified by (39), (40), (41), (42), (60), and (61) yeild $4 M+2$ algebraic equations connecting $4 M+2$ unknown coefficients. Setting the determinant of these coefficients equal to zero leads to an explicit equation to solve for the eigenvalues or modes $\nu$. To illustrate the form of this modal equation, the case of a homogeneous ionosphere is considered. Thus $M=1$ and the boundary conditions yield 6 linear equations in the coefficients $f_{1}, f_{2}, g_{1}, g_{2}, \overrightarrow{p_{1}}$, and $\overrightarrow{p_{1}^{\prime}}$. The determinant of these coefficients is written out explicitly in table 1. Setting this monster equal to zero vields a rather involved transcendental equation for the determination of the modes $\nu$. The size of the determinant grows linearly as the number of slabs is increased (i.e., $M>1$ ). In the case of any number of slabs, the determinant equation for the mode can be written in matrix form as follows: 
TABLE 1. Determinant of the coefficients for the case of homogeneous ionosphere

\begin{tabular}{|c|c|c|c|c|c|}
\hline$\hat{h}_{\nu}^{(1)}(k c)$ & $\hat{h}_{\nu}^{(2)}(k c)$ & 0 & 0 & $-e^{-u_{1} c}$ & $-e^{-u_{1} c}$ \\
\hline 0 & 0 & $\hat{h}_{\nu}(1)(k c)$ & $\hat{h}_{\nu}{ }^{(2)}(k c)$ & $-\alpha\left(u_{1}\right) e^{-u_{1} c}$ & $-\alpha\left(u_{1}^{\prime}\right) e^{-u_{1}^{\prime} c}$ \\
\hline 0 & 0 & $k \hat{h}_{\nu}^{(1)^{\prime}}(k c)$ & $k \hat{h}_{\nu}(2)^{\prime}(k c)$ & $u_{1} \alpha\left(u_{1}\right) e^{-u_{1} c}$ & $u_{1}^{\prime} \alpha\left(u_{1}^{\prime}\right) e^{-u_{1}^{\prime} c}$ \\
\hline$\frac{k}{\epsilon_{0}} \hat{h}_{\nu}^{(1)^{\prime}}(k c)$ & $\frac{k}{\epsilon_{0}} \hat{h}_{\nu^{(2)}}^{\prime}(k c)$ & 0 & 0 & $\frac{1}{\epsilon_{1}}\left[u_{1}+\alpha\left(u_{1}\right) g_{1} \omega\right] e^{-u_{1} c}$ & $\frac{1}{\epsilon_{1}}\left[u_{1}^{\prime}+\alpha\left(u_{1}^{\prime}\right) g_{1} \omega\right] e^{-u_{1}^{\prime} c}$ \\
\hline$\left[i \epsilon_{0} \omega Z_{g} \hat{h}_{\nu}^{(1)}(k a)\right.$ & {$\left[i \epsilon_{0} \nu Z_{g} \hat{h}_{\nu}^{(2)}(k a)\right.$} & 0 & 0 & 0 & 0 \\
\hline$\left.-k \hat{h}_{\nu}(1)^{\prime}(k a)\right]$ & $\left.-k \hat{h}_{\nu}^{(2)}{ }^{\prime}(k a)\right]$ & & & & \\
\hline 0 & 0 & {$\left[i \mu \omega Y_{g} \hat{h}_{\nu}^{(1)}(k a)\right.$} & {$\left[i \mu \omega Y_{g} \hat{h}_{\nu}^{(2)}(k a)\right.$} & 0 & 0 \\
\hline & & $\left.\left.-k \hat{h}_{\nu}^{(1)}\right)^{\prime}(k a)\right]$ & $\left.-k \hat{h}_{\nu}^{(2)}{ }^{\prime}(k a)\right]$ & & \\
\hline
\end{tabular}

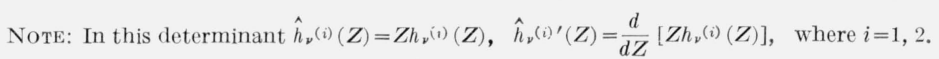

$$
\begin{aligned}
& \operatorname{det}\left\{\left[\begin{array}{ll}
R_{v} & 0 \\
0 & R_{h}
\end{array}\right]\left[\begin{array}{ll}
R_{\|} & \perp R_{\|} \\
R_{\perp} & { }_{\perp} R_{\perp}
\end{array}\right] \frac{h_{\nu}^{(1)}(k a) h_{\nu}^{(2)}(k c)}{h_{\nu}^{(2)}(k a) h_{\nu}^{(1)}(k c)}\right. \\
& \left.-\left[\begin{array}{ll}
1 & 0 \\
0 & 1
\end{array}\right]\right\}=0 \text {, }
\end{aligned}
$$

where the first two factors are matrix reflection coefficients. Explicitly,

and

$$
R_{v}=-\left[\frac{\ln ^{\prime} x h_{\nu}^{(1)}(x)-i Z_{g} / \eta_{0}}{\ln ^{\prime} x h_{\nu}^{(2)}(x)-i Z_{g} / \eta_{0}}\right]_{x=k a}
$$

$$
R_{h}=-\left[\frac{\ln ^{\prime} x h_{\nu}^{(1)}(x)-i Y_{g} \eta_{0}}{\ln ^{\prime} x h_{\nu}^{(2)}(x)-i Y_{g} \eta_{0}}\right]_{x=k a},
$$

where $\ln ^{\prime}$ is the logarithmic derivative defined by

$$
\ln ^{\prime} F(x)=\frac{d F(x) / d x}{F(x)} .
$$

$R_{\imath}$ and $R_{h}$ can be interpreted as ground-reflection coefficients for vertically and horizontally polarized waves, respectively. The explicit form of $R_{\|}$, ${ }_{\perp} R_{\|}$, etc., for the present problem could be written out in full. However, there is no need to do this as they have the same form as the reflection coefficient matrix for a plane stratified ionosphere. This aspect of the subject has been discussed recently in some detail [Wait, 1963]. To make direct use of numerical data already available for planar reflection coefficients, it is necessary to invoke the Debye approximations for the Hankel functions $h_{\nu}^{(1)}(k c)$ and $h_{\nu}^{(2)}(k c)$ and their derivatives. The restriction for this step is

$$
(k a / 2)^{1 / 3} C^{\prime}>>1,
$$

where $C^{\prime}$ is the (complex) cosine of the local angle of incidence at the ionosphere. In terms of $\nu$ and $k c$ it is not difficult to show that

$$
C^{\prime}=\left[1-\frac{(\nu+1 / 2)^{2}}{(k c)^{2}}\right]^{1 / 2} \text {. }
$$

In the theoretical model discussed in this paper, the earth's magnetic field was taken to be vertical everywhere. A rigorous treatment of the earthionosphere waveguide with a nonvertical magnetic field does not seem to be possible. However, an approximate approach to the subject, which utilizes the concept of surface impedances, also leads to a modal equation of the form of (65) above [Wait and Spies, 1960; Wait, 1961a, 1963].

\section{Final Discussion}

The particular problem discussed in this paper has also been considered by Johler and Berry [1962]. However, their solution for the anisotropic ionosphere does not agree with the one given in this paper and with a previous formulation of the author [Wait, 1961a] using a similar approach. It appears that the origin of the discrepancy is their assumption that the Hertz potentials $\Pi^{e}$ and $\Pi^{m}$ in the ionosphere are not coupled by the boundary conditions. ${ }^{1}$ This coupling is evidenced by the forms of (18) and (19) in the present paper. Furthermore, their Hertz potentials $\Pi^{e}$ and $\Pi^{m}$, which are related to the Debye potentials $N$ and $M$, are not solutions of uncoupled wave equations in the ionosphere.

The coupling of ordinary and extraordinary waves by the boundary conditions appears to be an essential point in the analysis. Physically this is important because the transmitted waves in the ionosphere are almost circularly polarized even though the waves in the space between the earth and the ionosphere are nearly linearly polarized.

\section{References}

Bremmer, H. (1949), Terrestrial radio waves (Elsevier Publishing Co., New York and Amsterdam).

Budden, K. G. (1961), Radio waves in the ionosphere (Cambridge University Press).

Budden, K. G. (1962), The wave-guide mode theory of wave propagation (Prentice-Hall, Englewood Cliffs, N.J.).

Johler, J. R., and L. A. Berry (Nov.-Dec. 1962), Propagation of terrestrial radio waves of long wavelength, J. Res. NBS 66D (Radio Prop.) No. 6, 737-772.

${ }^{1}$ It is also of interest to note that their general solution does not reduce directly to the well-known isotropic solution given by their equation (73) when the earth's magnetic field is removed. 
Katzin, M., and B. Y.-C. Koo (April 1962), Studies in ionospheric propagation, Pt. I, The exact earth-flattening procedure in ionospheric propagation problems, Final Report on Contract AF 19(604)-7233 for U.S. Air Force Cambridge Research Laboratories.

Krasnushkin, P. E. (July 1961), Propagation of long and very long radio waves around the earth and the $C, D$, and E layers of the ionosphere from the standpoint of information theory, Doklady Akademii Nauk. SSSR 139, 67-70.

Sen, H. K., and A. A. Wyler (Dec. 1960), On the generalization of the Appleton-Hartree magneto ionic formulas, J. Geophys. Res. 65, 3931-3950.

van der Wijck, C. Th. F. (1946), Thesis, University of Delft, Holland.

Wait, J. R. (Jan.-Feb. 1961a), A new approach to the mode theory of VLF propagation, J. Res. NBS 65D (Radio Prop.) No. 1, 37-46.

Wait, J. R. (Apr.-June 1961b), Some boundary value problems involving plasma media, J. Res. NBS 65B (Math. and Math. Phys.), No. 2, 137-150.

Wait, J. R. (1962), Electromagnetic waves in stratified media (Pergamon Press, Oxford).

Wait, J. R. (1963), The mode theory of VLF radio propagation for a spherical earth and a concentric anisotropic ionosphere, Can. J. Phys. 41, No. 2, 299-315.

Wait, J. R., and K. Spies (Aug. 1960), Influence of earth curvature and the terrestrial magnetic field on VLF propagation, J. Geophys. Res. 65, No. 8, 2325-2331.
Johler, J. R. (1962), On radio wave reflections at a continuously stratified plasma with collisions proportional to energy and magnetic induction, Proc. Conference on Ionosphere (Physical Society London) (in press).

\subsection{Additional References}

Budden, K. G., and P. C. Clemmow (1957), Coupled forms of the differential equations governing radio propagation in the ionosphere, 2, Proc. Cambridge Phil. Soc. 53, 669682.

Ferraro, A. J. (Sept. 1962), Multi-slab concept applied to radio-wave propagation in the ionosphere and its limit to the continuous ionosphere, J. Geophys. Res. 67, 3817-3822.

Ferraro, A. J., and J. J. Gibbons (1959), Polarization computations by means of the multi-slab approximation, J. Atmos. and Terrest. Phys. 16, 136-144.

Johler, J. R. (Apr. 1962), Propagation of the low-frequency radic signal, Proc. IRE 50, 404-427.

Crombie, D. D. (Sept.-Oct. 1961), Reflection from a sharply bounded ionosphere for VLF propagation perpendicular to the magnetic meridian, J. Res. NBS 65D (Radio Prop.) No. 5, 455-463.

(Paper 67D3-264) 